\title{
Integrative analysis of promising molecular biomarkers and pathways for coronary artery disease using WGCNA and MetaDE methods
}

\author{
SHILIN YAN \\ Department of Cardiology, Yangling Demonstration Zone Hospital, Xianyang, Shaanxi 712100, P.R. China
}

Received January 8, 2018; Accepted May 31, 2018

DOI: $10.3892 / \mathrm{mmr} .2018 .9277$

\begin{abstract}
The present study aimed to examine the molecular mechanisms of coronary artery disease (CAD). A total of four microarray datasets (training dataset no. GSE12288; validation dataset nos. GSE20680, GSE20681 and GSE42148) were downloaded from the Gene Expression Omnibus database, which included CAD and healthy samples. Weighted gene co-expression network analysis was applied to identify highly preserved modules across the four datasets. Differentially expressed genes (DEGs) with significant consistency in the four datasets were selected using the MetaDE method. The overlapping genes amongst the DEGs with significant consistency and in the preserved modules were used to construct a protein-protein interaction (PPI) network, followed by functional enrichment analysis. A total of 11 modules were established in the training dataset, and five of them were highly preserved across all four datasets, including 873 genes. There was a total of 836 DEGs with significant consistency in the four datasets. A total of 177 overlapping genes were selected, with which a PPI network was constructed. The top five genes of the PPI network were identified based on their degrees: LCK proto-oncogene, Src family tyrosine kinase $(L C K)$, euchromatic histone lysine methyltransferase 2 (EHMT2), inosine monophosphate dehydrogenase 2 (IMPDH2), protein phosphatase 4 catalytic subunit $(P P P 4 C)$ and $\zeta$-chain of T-cell receptor associated protein kinase $70(Z A P 70)$. Genes in the PPI network were significantly involved in a number of Kyoto Encyclopedia Genes and Genomes pathways, including the 'natural killer cell mediated cytotoxicity', 'primary immunodeficiency' and 'Fc gamma R-mediated phagocytosis' pathways. LCK, EHMT2, IMPDH2, PPP4C and ZAP70 are suggested as promising molecular biomarkers for CAD. The 'natural killer cell mediated cytotoxicity', 'primary immunodeficiency' and
\end{abstract}

Correspondence to: Dr Shilin Yan, Department of Cardiology, Yangling Demonstration Zone Hospital, Yangling Zone, 8 Houji Road, Xianyang, Shaanxi 712100, P.R. China

E-mail: yanshilin128@sina.com

Key words: weighted gene co-expression network, coronary artery disease, network, module, pathway, protein-protein interaction
'Fc gamma R-mediated phagocytosis' pathways may serve important roles in CAD.

\section{Introduction}

Coronary artery disease (CAD) remains one of the most common causes of morbidity and mortality globally, with an increased prevalence predicted in the near future (1). The occurrence of CAD events is primarily due to crosstalk between genetic and environmental factors (2). A number of risk factors of CAD have been identified, including smoking, obesity and family history (3). Available treatment options primarily include lifestyle alterations, medical treatment and surgical interventions, which are chosen depending on comorbidities and the preferences of the individual patient (4). Prevention and treatment of this disease remains a daunting task. Therefore, further research is required to elucidate the underlying biological features for the development of more adequate therapy for patients.

The rapid prevalence of high-throughput microarray technologies has facilitated identification of genome variations in diseases, contributing to a deeper understanding of pathogenesis and the development of promising biomarkers $(5,6)$. Such technologies have been applied to investigate the underlying mechanisms of CAD. Ren et al (7) identified a circulating micro (mi)RNA signature for CAD using co-expression network analyses on miRNA array data. In addition, a study of genomic DNA methylation profiling in patients with CAD was conducted (8). Furthermore, Liu et al (9) performed a secondary analysis using a weighted gene co-expression network (WGCNA) on microarray data of CAD samples (dataset no. GSE23561), which were downloaded from the Gene Expression Omnibus (GEO) database. It was identified that the glucose-6-phosphate 1-dehydrogenase, protein S100-A7 and hypertrophic cardiomyopathy pathways were involved in CAD (9). However, this previous study had the limitation of sample size (six CAD samples and nine normal samples).

Therefore, four microarray datasets of CAD from the GEO database were included in the present study. WGCNA was used to construct a co-expression network and the highly preserved modules in the four datasets were determined. The MetaDE method in $\mathrm{R}$ language, which is capable of conducting 12 primary meta-analysis methods (10), has been utilized for 
the detection of differentially expressed genes (DEGs) in a number of diseases, including gastric cancer (11) and colorectal cancer (12). In the present study, the DEGs with significant consistency across all four datasets were selected using the MetaDE method and subsequently compared with the genes in the highly preserved WGCNA modules. Subsequently, the overlapping genes were used to construct a protein-protein interaction (PPI) network, followed by functional analysis of the genes in the network. The in-depth analysis conducted in the present study may provide novel insights into the pathogenesis of CAD.

\section{Materials and methods}

Microarray data. Microarray data for CAD were searched in NCBI GEO (http://www.ncbi.nlm.nih.gov/geo/). Inclusion criteria were: i) The dataset belonged to a gene expression profile; ii) samples in the dataset were collected from blood; iii) the samples included patients and healthy controls; iv) the dataset was based on human gene expression profiles; and v) the sample number was $\geq 20$. A total of four datasets, GSE12288 ( $\mathrm{n}=222$; CAD, 112; control, 110) (13), GSE20680 ( $\mathrm{n}=139 ;$ CAD, 87; control, 52) (14), GSE20681 ( $\mathrm{n}=198$; CAD, 99; control, 99) (15) and GSE42148 (n=24; CAD, 13; control, 11; https://www.ncbi.nlm.nih.gov/geo/query/acc .cgi?acc=GSE42148) that met the criteria were included in the present study (Table I).

Data preprocessing. Raw data in CEL files from GSE12288 were downloaded from the Affy platform (https://www .ncbi.nlm.nih.gov/geo/query/acc.cgi?acc=GPL96) and subsequently converted into gene symbols under pretreatment. The median method was used to fill in the missing values, the MicroArray Suite method to complete background correction, and the quantiles method to normalize the data, using oligo 1.41.1 in R3.3.1 language (http://www.bioconductor .org/packages/release/bioc/html/oligo.html) (16). GSE20680, GSE20681 and GSE42148 were downloaded from the Agilent platform (Agilent Technologies, Inc., Santa Clara, CA, USA). Microarray raw data (TXT files) of the three datasets were log2-transformed using Limma 3.34.0 (https://bioconductor .org/packages/release/bioc/html/limma.html) to achieve an approximate normal distribution, and were subsequently standardized using the median normalization method (17).

WGCNA analysis. WGCNA is a well-established method for constructing scale-free gene co-expression networks, which is characterized by the use of soft thresholding $(18,19)$. GSE12288 was used as the training dataset, while GSE20680, GSE20681 and GSE42148 were the validations sets. Genes with coefficients of variation $<0.1$ were discarded. Correlations between the gene expression in the four datasets were evaluated with the verboseScatterplot function of the WGCNA package (https://labs.genetics.ucla .edu/horvath/CoexpressionNetwork/Rpackages/WGCNA/). Weighted gene co-expression networks of the genes in the training dataset were constructed using the WGCNA method as previously described (20-22). First, the soft threshold power of $\beta$ was set as 18 (scale-free $\mathrm{R}^{2}=0.9$ ), according to the scale free topology criterion. The weighted adjacency matrix was subsequently constructed. Adjacencies and correlations were transformed into a topological overlap matrix (TOM), followed by calculating the corresponding dissimilarity (1-TOM). Subsequently, a hierarchical clustering analysis (23) of genes was performed using 1-TOM as the distance measure. Modules were detected using dynamic tree cut algorithm with a minimum module size of 50 and a minimum cut height of 0.95 . Furthermore, module preservation between the training set and the three validation sets was measured using the module preservation function of the WGCNA software package. Possible functions of the preserved module were studied using the user List Enrichment function of the WGCNA package.

IdentificationofDEGswithsignificantconsistencyinfourdatasets. DEGs between CAD samples and healthy controls were screened in each of the above four datasets using the metaDEmethod $(10,24)$ in R language (https://cran.r-project.org/web/packages/MetaDE/). Heterogeneity was examined across the four datasets to assess the consistency of gene expression, by calculating tau 2 and Qpval values. A tau2=0 and Qpval $>0.05$ indicated that the gene was homogeneous and unbiased. The thresholds for DEG identification were tau2=0, Qpval $>0.05$ and false discovery rate $<0.05$. Consistency of the DEGs was detected using the MetaDE method, and the DEGs with significant consistency in the four datasets were selected for further analysis.

Construction of a PPI network. A PPI network was constructed to evaluate the interactions between genes in the above network. The common genes in the preserved modules that were obtained from WGCNA, and the DEGs with significant consistency, were selected to construct a PPI network based on three databases: The Biological General Repository for Interaction Datasets 3.4.153 (http://thebiogrid.org/) (25); the Human Protein Reference Database Release 9 (http://www.hprd.org/) (26); and STRING 10.5 (https://string-db.org/) (27). The PPIs revealed in at least two of the three databases were extracted for the PPI network, visualized using Cytoscape 3.3 software (http://www .cytoscape.org/) (28). In the PPI network, a node represents a gene; the undirected link between two nodes is an edge, denoting the interaction between two genes; and the degree of a node corresponds to the number of interactions of a gene with other genes in the network.

Gene ontology (GO) functional and kyoto encyclopedia of genes and genomes (KEGG) pathway enrichment analyses. In order to elucidate the possible biological roles of the genes in the PPI network, GO (29) functional and KEGG (30) pathway enrichment analyses were performed using the Database for Annotation, Visualization and Integrated Discovery 6.8 software (31). GO terms have three categories, including biological process (BP), cellular compartment and molecular function. $\mathrm{P}<0.05$ was considered to indicate a significant difference for GO terms and KEGG pathways.

\section{Results}

Identification of key WGCNA modules. Following data preprocessing for the four datasets, the present study attempted 
Table I. Information on the four Gene Expression Omnibus datasets.

\begin{tabular}{llcrrr}
\hline Accession no. & Platform & Probe no. & Total sample no. & CAD & Control \\
\hline GSE12288 & GPL96 & 22283 & 222 & 112 \\
GSE20680 & GPL4133 & 45220 & 139 & 87 & 52 \\
GSE20681 & GPL4133 & 45220 & 198 & 99 \\
GSE42148 & GPL13607 & 62976 & 24 & 13 \\
\hline
\end{tabular}

CAD, coronary artery disease.
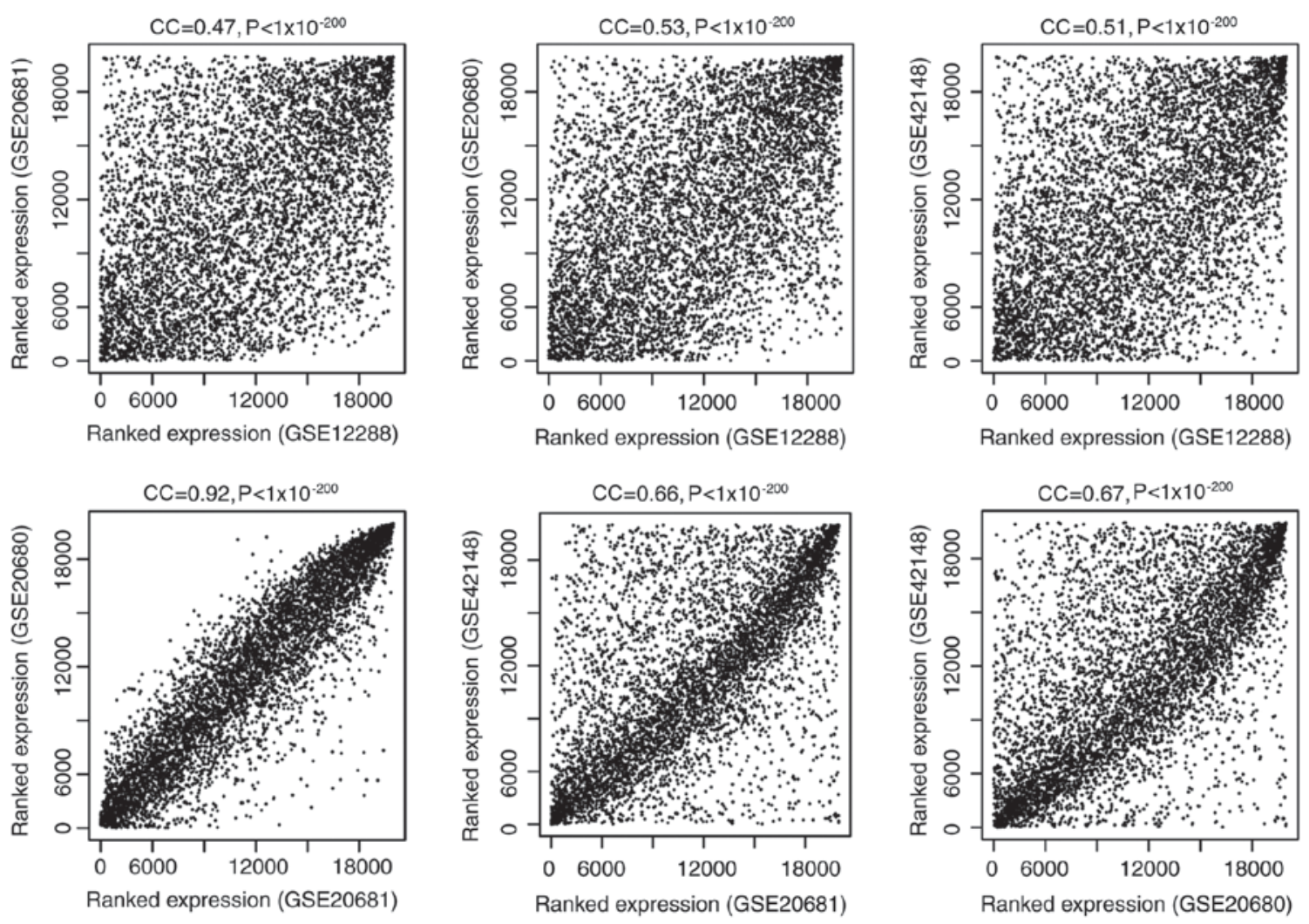

Figure 1. Correlation analysis of genes in four datasets from the Gene Expression Omnibus database. CC, correlation coefficient.

to identify CAD-associated co-expression modules using WGCNA. Using the aforementioned methods, the correlation coefficients (CCs) of genes in the four datasets were obtained, and the CCs were 0.47-0.92 with P-values $<1 \times 10^{-200}$ between any two datasets (Fig. 1), suggesting a good consistency of the common genes across all datasets.

As a result, 11 co-expression modules were identified for genes in the training set using WGCNA (Fig. 2A). These modules are illustrated in branches of the dendrogram with different colors. To evaluate the robustness of these modules in the training set, the modules were re-constructed in the three validation datasets, GSE20680, GSE20681 and GSE42148, separately (Fig. 2B-D). Genes in the three validation sets were colored, according to the module color in the training set. Multi-dimensional scaling of expression data of all genes in these modules demonstrated that genes in the same module appeared to cluster together and exhibited a similar expression pattern (Fig. 3A). Additionally, hierarchical clustering analysis of these modules in the four datasets revealed that modules of the same branch tended to have similar gene expression patterns (Fig. 3B).

In total, five of the 11 modules, black, brown, magenta, turquoise and yellow modules, with Z-scores $>5$ were determined to be well preserved across the four datasets, including 873 genes (Table II). This suggested that the five highly preserved modules may be closely associated with CAD. Of the five modules, genes in the black module were significantly linked to 'response to glucocorticoid stimulus'; those in the brown module were significantly associated with 'regulation of transcription'; and those in the magenta module may be involved in 'protein localization' (Table II). Notably, the turquoise and the yellow module exhibited functions in 'immune response' (Table II).

Screening for DEGs with significant consistency in the four datasets. Using the metaDE method, 836 DEGs were 
A

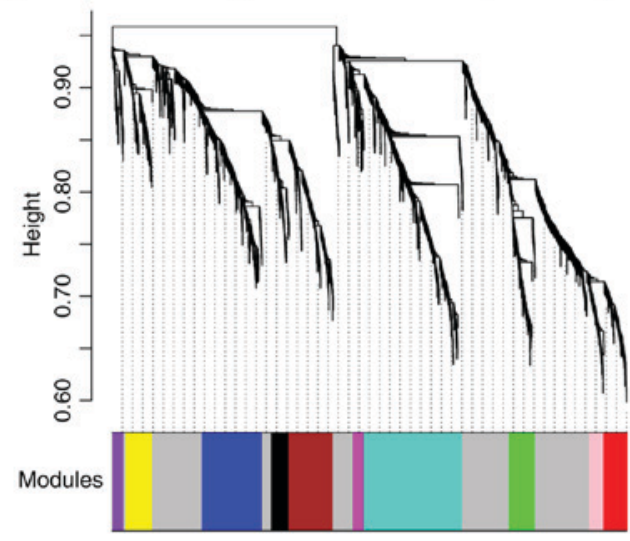

C

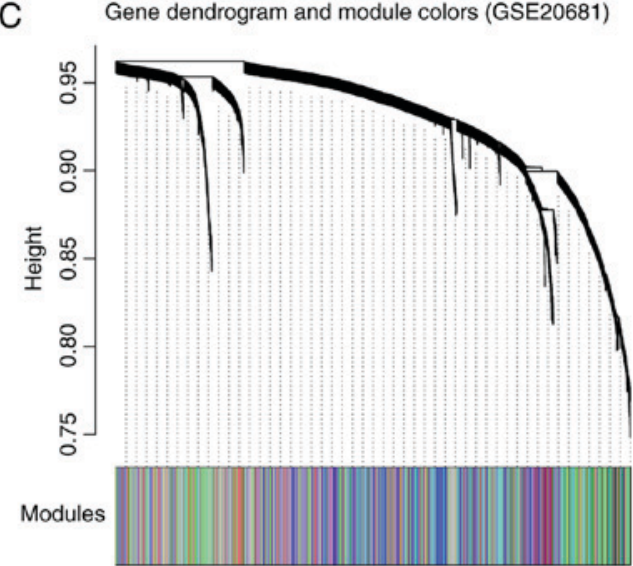

B Gene dendrogram and module colors (GSE20680)

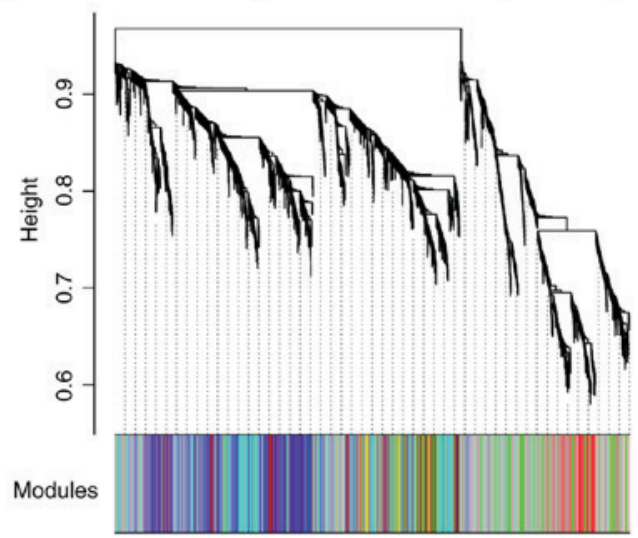

D

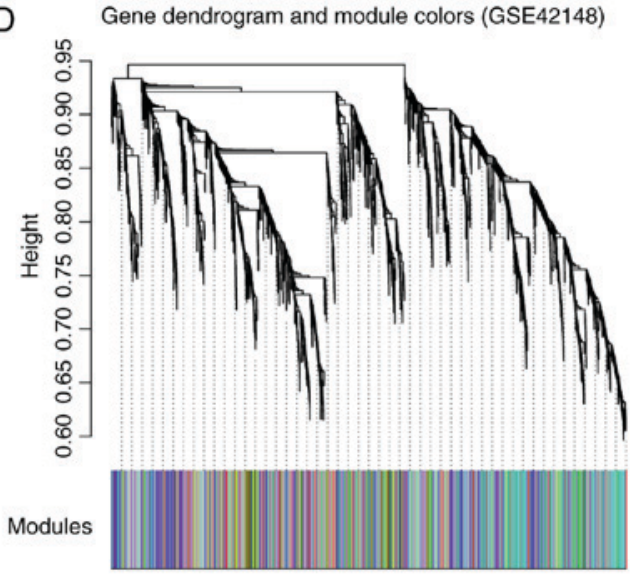

Figure 2. Identification of gene co-expression modules. (A) Training set GSE12288, and three validation sets, (B) GSE20680, (C) GSE20681 and (D) GSE42148, were examined. Modules are marked in different colors.

A

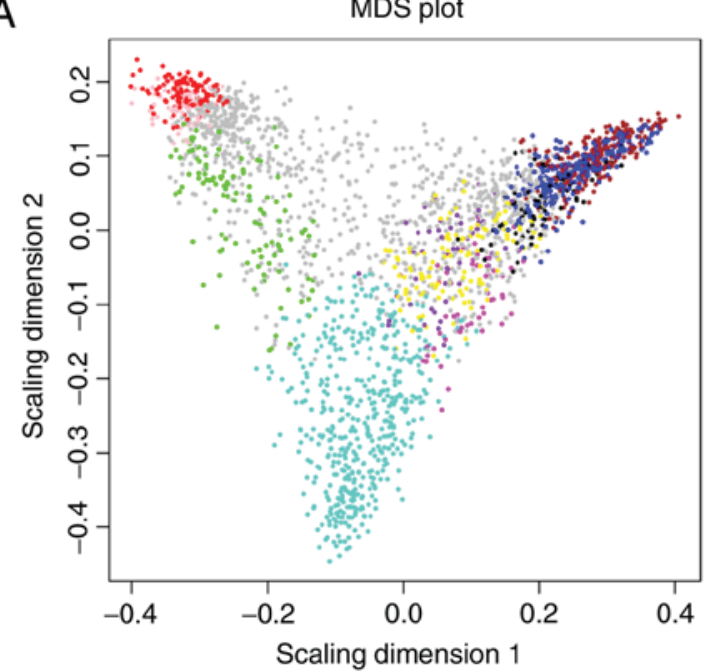

B

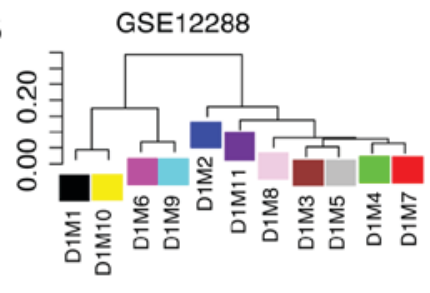

GSE20680

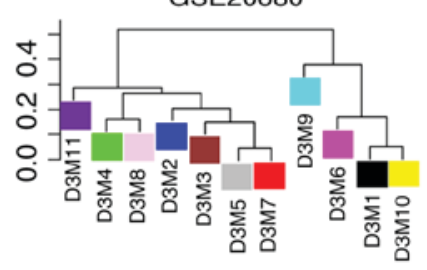

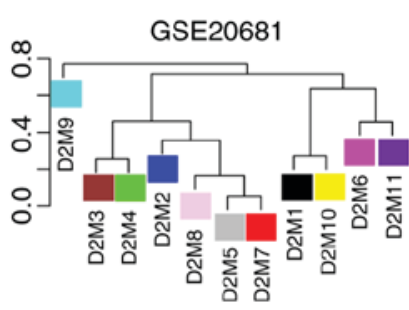

GSE42148

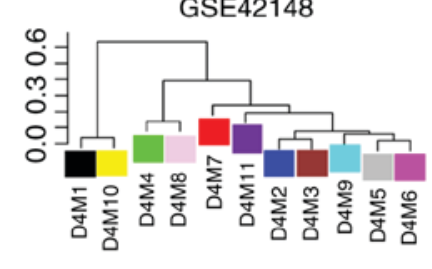

Figure 3. MDS plot and hierarchical dendrograms of the four datasets. (A) MDS plot of expression data of genes in 11 modules. (B) Hierarchical clustering tree of 11 modules in the four datasets. Modules are denoted via a 'D' and 'M' system: D1, GSE12288; D2, GSE20681; D3, GSE20680; D4, GSE42148; M1, black; M2, dark blue; M3, brown; M4, green; M5, grey; M6, magenta; M7, red; M8, pink; M9, turquoise; M10, yellow; and M11, purple. MDS, multi-dimensional scaling.

identified with significant consistency across the four datasets. A heatmap for these DEGs demonstrated that the expression patterns of these DEGs differed between the CAD and control samples (Fig. 4).
Construction of a PPI network for the overlapping genes. A total of 177 genes were overlapping between WGCNA modules and the DEGs with significant consistency (Fig. 5A). Of them, 92 genes were included in the turquoise module, 26 in the 
Table II. Characteristics of weighted gene co-expression network modules.

\begin{tabular}{|c|c|c|c|c|c|c|c|}
\hline \multicolumn{4}{|c|}{ Dataset } & \multicolumn{4}{|r|}{ Characteristic } \\
\hline GSE12288 & GSE20681 & GSE20680 & GSE42148 & Color & Size & Z-score & Module characterization \\
\hline D1M1 & D2M1 & D3M1 & D4M1 & Black & 85 & 13.81 & Response to glucocorticoid stimulus \\
\hline D1M2 & $\mathrm{D} 2 \mathrm{M} 2$ & D3M2 & D4M2 & Blue & 285 & 0.58 & Humoral immune response \\
\hline D1M3 & D2M3 & D3M3 & D4M3 & Brown & 211 & 5.26 & Regulation of transcription \\
\hline D1M4 & D2M4 & D3M4 & D4M4 & Green & 121 & 0.16 & Regulation of transcription \\
\hline D1M5 & D2M5 & D3M5 & D4M5 & Grey & 572 & 3.92 & Defense response \\
\hline D1M6 & D2M6 & D3M6 & D4M6 & Magenta & 51 & 5.66 & Protein localization \\
\hline D1M7 & D2M7 & D3M7 & D4M7 & Red & 68 & 0.63 & Cell surface receptor linked signal transduction \\
\hline D1M8 & D2M8 & D3M8 & D4M8 & Pink & 108 & 1.31 & Second-messenger-mediated signaling \\
\hline D1M9 & D2M9 & D3M9 & D4M9 & Turquoise & 400 & 14.34 & Immune response \\
\hline D1M10 & D2M10 & D3M10 & D4M10 & Yellow & 126 & 12.54 & Immune response \\
\hline D1M11 & D2M11 & D3M11 & D4M11 & Purple & 50 & 1.35 & Lymphocyte activation \\
\hline
\end{tabular}

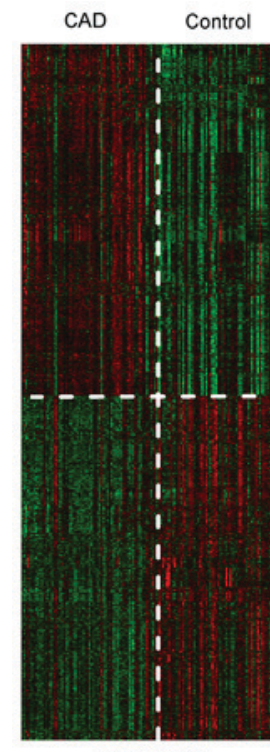

GSE12288

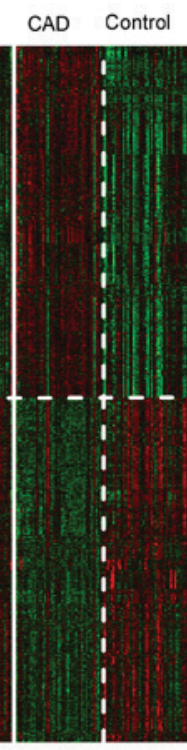

GSE20681

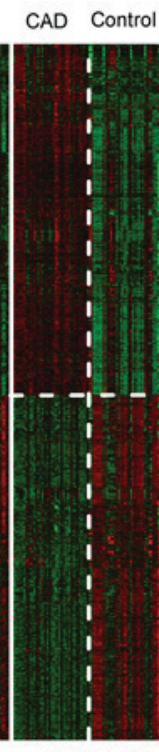

GSE20680

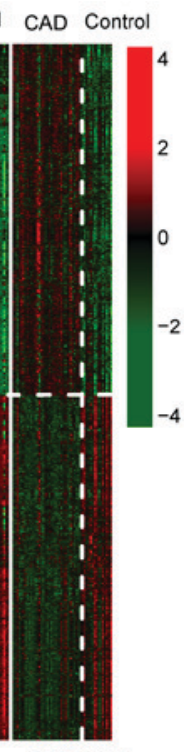

GSE42148
Figure 4. Heatmap of hierarchical clustering of differentially expressed genes with significant consistency, identified using the metaDE method. CAD and normal samples notably differed from each other with respect to gene expression patterns. CAD, coronary artery disease.

yellow module, 16 in the black module, 31 in the brown module and 12 in the magenta module (Fig. 5B). Based on the three databases mentioned above, a PPI network was built with these overlapping genes. In total, 150 paired PPIs that appeared in at least two of the three databases were included in the PPI network (Fig. 6A), which contained 59 downregulated genes and 40 upregulated genes (Fig. 6B). All genes in the network were ranked in a descending order, according to their degrees. The top five genes were LCK proto-oncogene, Src family tyrosine kinase ( $L C K$; degree $=15)$, euchromatic histone lysine methyltransferase $2(E H M T 2$; degree $=14)$, inosine monophosphate dehydrogenase $2(I M P D H 2$; degree $=12)$, protein phosphatase 4 catalytic subunit $(P P P 4 C$; degree $=11)$ and $\xi$-chain of T-cell receptor associated protein kinase $70(Z A P 70$; degree $=11)$.
Functional annotation. The enrichment analysis indicated that genes in the PPI network were significantly associated with numerous GO BP terms, including the 'transmembrane receptor protein tyrosine kinase signaling pathway' and 'peptidyl-tyrosine autophosphorylation' (Table III). With regard to KEGG pathways, there were five significant pathways, including 'metabolic pathways', 'natural killer cell mediated cytotoxicity', 'fructose and mannose metabolism', 'primary immunodeficiency', and 'Fc gamma R-mediated phagocytosis' (Table IV). Notably, 'natural killer cell mediated cytotoxicity' and 'primary immunodeficiency' pathways were significantly enriched with $L C K$ and ZAP70.

\section{Discussion}

CAD remains a primary public health concern (32). The present study attempted to dissect the underlying pathogenic mechanisms of CAD via a combined analysis of four GEO datasets, which contained CAD samples and healthy control samples. A total of 11 modules were detected in the WGCNA network, five of which were highly preserved across all datasets. Using the metaDE method, 836 common DEGs in the four datasets were identified. Furthermore, a PPI network was constructed with the 177 overlapping genes of the DEGs with significant consistency and the five preserved WGCNA modules. According to degree, the top five genes of the PPI network were LCK, EHMT2, IMPDH2, PPP4C and ZAP70. Notably, multiple significant pathways for genes in the PPI network were identified, including 'natural killer cell mediated cytotoxicity', 'primary immunodeficiency', and 'Fc gamma R-mediated phagocytosis' pathways.

The LCK protein encoded by the gene $L C K$, additionally termed lymphocyte-specific protein tyrosine kinase, is a member of the Src family of tyrosine kinases, which are involved in $\mathrm{T}$ cell signaling (33). Insufficient deactivation of LCK has been demonstrated to render patients with acute coronary syndrome (ACS) vulnerable to abnormal $\mathrm{T}$ cell responses (34). The ZAP-70 gene encodes the ZAP-70 enzyme, which belongs to the protein-tyrosine kinase family and is a 
A

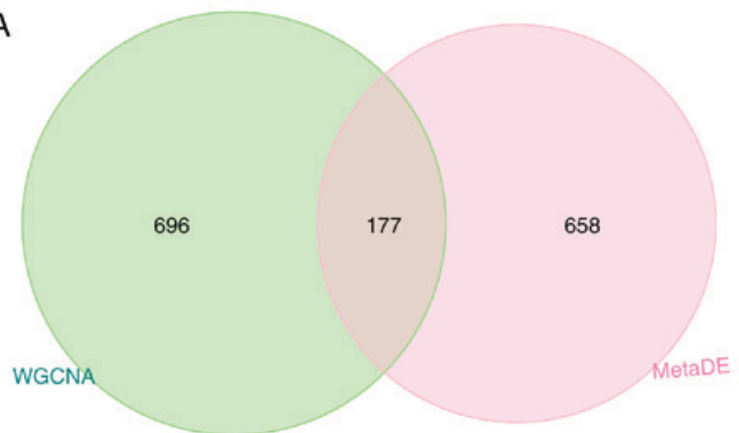

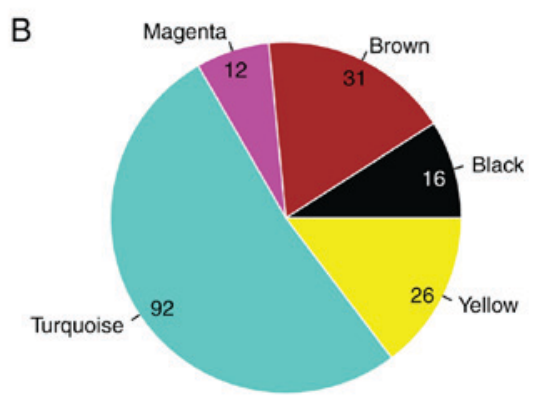

Figure 5. Analysis of overlapping genes. (A) Venn diagram of the overlapping genes between WGCNA modules (green) and the genes with significant consistency identified using MetaDE (pink). (B) Numbers of overlapping genes in each of the five highly preserved WGCNA modules. WGCNA, weighted gene co-expression network.
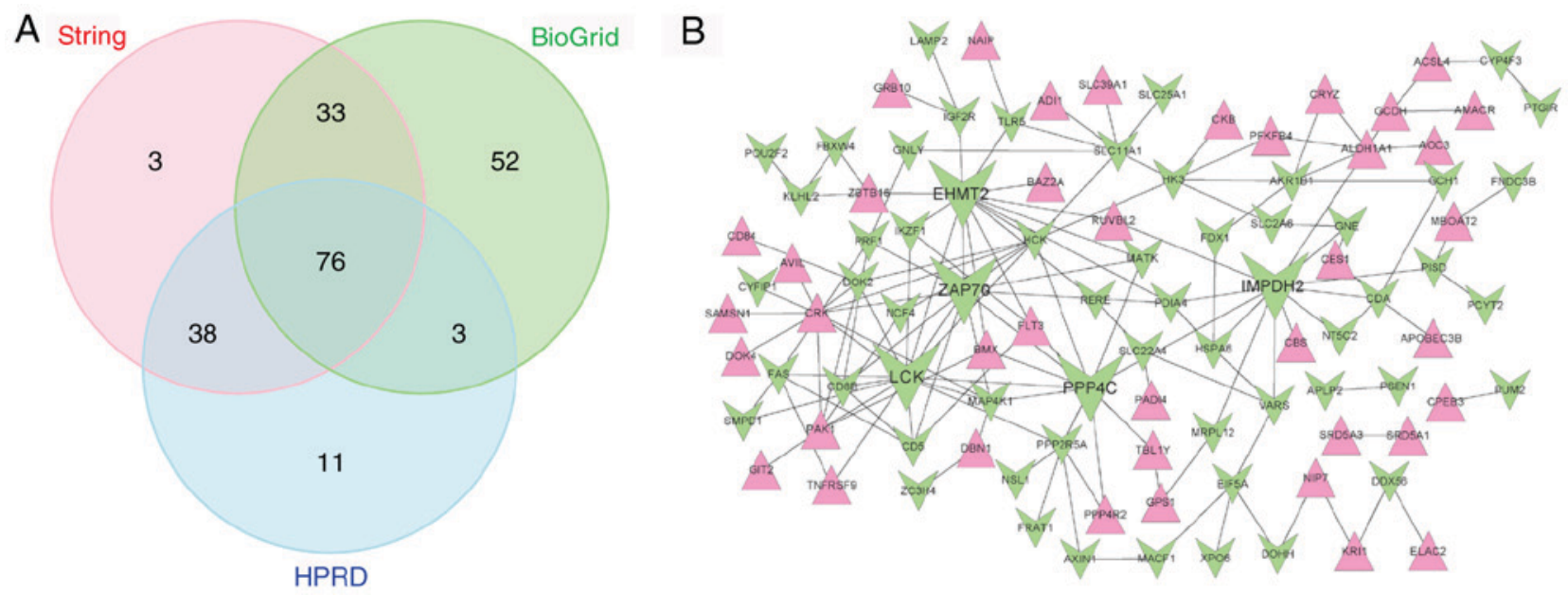

Figure 6. Construction of the PPI network. (A) Venn diagram of PPIs obtained from BioGRID, HPRD Release 9 and STRING. (B) PPI network. A red triangle denotes an upregulated gene and a green inverted triangle denotes a downregulated gene. The top five genes with higher degrees compared with the other genes in the network are represented by five extra-large green inverted triangles, separately. PPI, protein-protein interaction; BioGRID, Biological General Repository for Interaction Datasets; HPRD, Human Protein Reference Database; LCK, LCK proto-oncogene, Src family tyrosine kinase; EHMT2, euchromatic histone lysine methyltransferase 2; IMPDH2, inosine monophosphate dehydrogenase 2; PPP4C, protein phosphatase 4 catalytic subunit; ZAP70, $\zeta$-chain of T-cell receptor associated protein kinase 70 .

T cell receptor. In T cell signaling, ZAP-70 binds to the CD3 complex that is phosphorylated by the LCK protein (35). It has been established that the ZAP-70 protein expression is able to act as a marker for chronic lymphocytic leukemia or small lymphocytic lymphoma (36).

In the present study, $L C K$ (degree=15) and ZAP70 (degree $=11$ ) were highlighted in the PPI network. Furthermore, based on the KEGG pathway enrichment analysis, $L C K$ and ZAP70 were significantly associated with 'natural killer cell mediated cytotoxicity' and 'primary immunodeficiency' pathways, which were associated with immune processes. Similarly, there is evidence that dysregulated adaptive immunity serves a causative role in ACS (37). Therefore, it may be inferred that $L C K$ and ZAP70 serve a role in CAD, which may partly be by regulating natural killer cell mediated cytotoxicity and primary immunodeficiency pathways. Additionally, a previous study with a novel knowledge-based approach revealed that the Fc gamma R-mediated phagocytosis pathway is a pathogenic mechanism for CAD (38). Likewise, the present study suggests that 'Fc gamma R-mediated phagocytosis' may have a function in CAD.
EHMT2, encoded by the gene EHMT2, termed G9a, is a histone methyltransferase that serves a critical role in epigenetic regulation within the nucleus accumbens (39). Histone methylation has emerged as a crucial epigenetic mechanism for cardiovascular development and homeostasis (40). Papait et al (41) demonstrated that EHMT2 orchestrates important epigenetic alterations in cardiomyocyte homeostasis and hypertrophy. Furthermore, EHMT1/2 has been suggested to be a therapeutic target against pathological cardiac hypertrophy (42). These results are in accordance with the present results, which demonstrated that EHMT2 was a predominant gene in the PPI network (degree=14). These collectively suggest a potentially critical role of EHMT2 in CAD.

IMPDH2, encoded by gene IMPDH2, termed IMP dehydrogenase 2 , acts as a rate-limiting enzyme in guanine nucleotide biosynthesis (43). It has been reported to be involved in a number of cancer types, including primary nasopharyngeal carcinoma (44) and prostate cancer (45). Nonetheless, the role of IMPDH 2 in CAD has been poorly defined. The PPP4C gene encodes the PP4C protein, which is a member of the type 2 serine/threonine protein phosphatase family (46), and partly 
Table III. Significant GO terms.

\begin{tabular}{|c|c|c|c|}
\hline Category & Term & Count of genes & P-value \\
\hline \multirow[t]{17}{*}{ Biology process } & Transmembrane receptor protein tyrosine kinase signaling pathway & 9 & $<0.001$ \\
\hline & Peptidyl-tyrosine autophosphorylation & 5 & $<0.001$ \\
\hline & Oxidation-reduction process & 13 & $<0.001$ \\
\hline & Inflammatory response & 10 & $<0.001$ \\
\hline & Mesoderm development & 4 & $<0.001$ \\
\hline & Innate immune response & 9 & 0.003 \\
\hline & Carbohydrate phosphorylation & 3 & 0.007 \\
\hline & Regulation of defense response to virus by virus & 3 & 0.011 \\
\hline & Chromatin remodeling & 4 & 0.013 \\
\hline & Response to lipopolysaccharide & 5 & 0.014 \\
\hline & Protein autophosphorylation & 5 & 0.017 \\
\hline & Peptidyl-lysine modification to peptidyl-hypusine & 2 & 0.022 \\
\hline & Lymphocyte proliferation & 2 & 0.028 \\
\hline & Fc-gamma receptor signaling pathway involved in phagocytosis & 4 & 0.035 \\
\hline & Response to fungicide & 2 & 0.039 \\
\hline & Negative regulation of $\mathrm{B}$ cell activation & 2 & 0.039 \\
\hline & Apoptotic process & 8 & 0.042 \\
\hline \multirow[t]{4}{*}{ Cellular component } & Extrinsic component of cytoplasmic side of plasma membrane & 5 & 0.000 \\
\hline & Ruffle & 4 & 0.012 \\
\hline & Protein phosphatase 4 complex & 2 & 0.021 \\
\hline & Neuron projection & 5 & 0.038 \\
\hline \multirow[t]{11}{*}{ Molecular function } & Protein tyrosine kinase activity & 5 & 0.007 \\
\hline & Kinase activity & 6 & 0.013 \\
\hline & Insulin receptor binding & 3 & 0.014 \\
\hline & Electron carrier activity & 4 & 0.015 \\
\hline & Cholestenone 5-alpha-reductase activity & 2 & 0.017 \\
\hline & Catalytic activity & 5 & 0.023 \\
\hline & Poly(A) RNA binding & 13 & 0.028 \\
\hline & Oxidoreductase activity & 5 & 0.028 \\
\hline & mRNA 3'-untranslated region binding & 3 & 0.032 \\
\hline & Receptor activity & 5 & 0.037 \\
\hline & Enzyme binding & 6 & 0.043 \\
\hline
\end{tabular}

Count of genes represents the number of genes significantly enriched in each GO term. GO, gene ontology.

Table IV. Significant KEGG signaling pathways.

\begin{tabular}{lccl}
\hline Term & Count & P-value & \\
\hline Metabolic pathways & 22 & 0.001 & $\begin{array}{l}\text { GCDH, CES1, GNE, AMACR, SPHK1, PISD, CKB, } \\
\text { GCH1, ALDH1A1, ADI1, HK3, NT5C2, AKR1B1, } \\
\end{array}$ \\
& & & $\begin{array}{l}\text { SMPD1, MBOAT2, CDA, CYP4F3, ACSL4, PCYT2, } \\
\text { IMPDH2, CBS, AOC3 }\end{array}$ \\
Natural killer cell mediated cytotoxicity & 5 & 0.022 & PRF1, LCK, ZAP70, PAK1, FAS \\
$\begin{array}{l}\text { Fructose and mannose metabolism } \\
\text { Primary immunodeficiency }\end{array}$ & 3 & 0.032 & PFKFB4, HK3, AKR1B1 \\
Fc gamma R-mediated phagocytosis & 3 & 0.036 & CD8B, LCK, ZAP70 \\
& 4 & 0.038 & HCK, SPHK1, PAK1, CRK
\end{tabular}

Count of genes denotes the number of genes significantly associated with each KEGG pathway. KEGG, Kyoto Encyclopedia of Genes and Genomes. 
regulates the phosphorylation of numerous proteins implicated in cardiac physiology and hypertrophy $(47,48)$. The present study indicated that $I M P D H 2$ and $P P P 4 C$ may be important genes in CAD.

Although a comprehensive bioinformatics analysis using four datasets was performed, there were a number of limitations. The potentially important genes associated with CAD lacked gene expression and functional validation. Further studies are required with substantial experiments in vivo and in vitro. Furthermore, the four datasets were not from the same platform, which may cause numerous deviations. Nevertheless, the present study provides novel insight into CAD pathogenesis.

In conclusion, the present study revealed that $L C K$, EHMT2, IMPDH2, PPP4C and ZAP70 may be proposed as genetic biomarkers for CAD. They may function via the involvement of the 'natural killer cell mediated cytotoxicity', 'primary immunodeficiency', and 'Fc gamma R-mediated phagocytosis pathways' in CAD. These results require further validation with substantial experiments.

\section{Acknowledgements}

Not applicable.

\section{Funding}

No funding was received.

\section{Availability of data and materials}

The datasets used and/or analyzed during the current study are available from the corresponding author on reasonable request.

\section{Authors' contributions}

SY performed data analyses, wrote the manuscript, conceived and designed the study.

\section{Ethics approval and consent to participate}

In the original article of the datasets, the trials were approved by the local institutional review boards of all participating centers and informed consent was obtained from all patients.

\section{Patient consent for publication}

Not applicable.

\section{Competing interests}

The authors declare that they have no competing interests.

\section{References}

1. Ohira $\mathrm{T}$ and Iso H: Cardiovascular disease epidemiology in Asia: An overview. Circ J 77: 1646-1652, 2013.

2. Sayols-Baixeras S, Lluís-Ganella C, Lucas G and Elosua R: Pathogenesis of coronary artery disease: Focus on genetic risk factors and identification of genetic variants. Appl Clin Genet 7: 15-32, 2014.

3. Torpy JM, Burke AE and Glass RM: Coronary heart disease risk factors. JAMA 302: 2388, 2009.
4. Hanson MA, Fareed MT, Argenio SL, Agunwamba AO and Hanson TR: Coronary artery disease. Prim Care 40: 1-16, 2013.

5. Callaway JL, Shaffer LG, Chitty LS, Rosenfeld JA and Crolla JA: The clinical utility of microarray technologies applied to prenatal cytogenetics in the presence of a normal conventional karyotype: A review of the literature. Prenat Diagn 33: 1119-1133, 2013.

6. Abulwerdi FA and Schneekloth JS Jr: Microarray-based technologies for the discovery of selective, RNA-binding molecules. Methods 103: 188-195, 2016.

7. Ren J, Zhang J, Xu N, Han G, Geng Q, Song J, Li S, Zhao J and Chen H: Signature of circulating microRNAs as potential biomarkers in vulnerable coronary artery disease. PLoS One 8: e80738, 2013

8. Sharma P, Garg G, Kumar A, Mohammad F, Kumar SR, Tanwar VS, Sati S, Sharma A, Karthikeyan G, Brahmachari V and Sengupta S: Genome wide DNA methylation profiling for epigenetic alteration in coronary artery disease patients. Gene 541: 31-40, 2014.

9. Liu J, Jing L and Tu X: Weighted gene co-expression network analysis identifies specific modules and hub genes related to coronary artery disease. BMC Cardiovasc Disord 16: 54, 2016.

10. Wang X, Kang DD, Shen K, Song C, Lu S, Chang LC, Liao SG, Huo Z, Tang S, Ding Y, et al: An R package suite for microarray meta-analysis in quality control, differentially expressed gene analysis and pathway enrichment detection. Bioinformatics 28: 2534-2536, 2012.

11. Cai H, Xu J, Han Y, Lu Z, Han T, Ding Y and Ma L: Integrated miRNA-risk gene-pathway pair network analysis provides prognostic biomarkers for gastric cancer. Onco Targets Ther 9: 2975-2986, 2016.

12. Qi C, Hong L, Cheng Z and Yin Q: Identification of metastasis-associated genes in colorectal cancer using metaDE and survival analysis. Oncol Lett 11: 568-574, 2016.

13. Sinnaeve PR, Donahue MP, Grass P, Seo D, Vonderscher J, Chibout SD, Kraus WE, Sketch M Jr, Nelson C, Ginsburg GS, et al: Gene expression patterns in peripheral blood correlate with the extent of coronary artery disease. PLoS One 4: e7037, 2009.

14. Elashoff MR, Wingrove JA, Beineke P, Daniels SE, Tingley WG, Rosenberg S, Voros S, Kraus WE, Ginsburg GS, Schwartz RS, et al: Development of a blood-based gene expression algorithm for assessment of obstructive coronary artery disease in non-diabetic patients. BMC Med Genomics 4: 26 , 2011.

15. Beineke P, Fitch K, Tao H, Elashoff MR, Rosenberg S, Kraus WE and Wingrove JA; PREDICT Investigators: A whole blood gene expression-based signature for smoking status. BMC Med Genomics 5: 58, 2012

16. Parrish RS and Spencer HJ III: Effect of normalization on significance testing for oligonucleotide microarrays. J Biopharm Stat 14: 575-589, 2004.

17. Ritchie ME, Phipson B, Wu D, Hu Y, Law CW, Shi W and Smyth GK: Limma powers differential expression analyses for RNA-sequencing and microarray studies. Nucleic Acids Res 43: e47, 2015 .

18. Langfelder P and Horvath S: WGCNA: An R package for weighted correlation network analysis. BMC Bioinformatics 9: $559,2008$.

19. Zhang B and Horvath S: A general framework for weighted gene co-expression network analysis. Stat Appl Genet Mol Biol 4: Article17, 2005.

20. Zhai X, Xue Q, Liu Q, Guo Y and Chen Z: Colon cancer recurrence-associated genes revealed by WGCNA co-expression network analysis. Mol Med Rep 16: 6499-6505, 2017.

21. Vilne B, Skogsberg J, Foroughi Asl H, Talukdar HA, Kessler T, Björkegren JLM and Schunkert H: Network analysis reveals a causal role of mitochondrial gene activity in atherosclerotic lesion formation. Atherosclerosis 267: 39-48, 2017.

22. Min S, Sun T, He Z and Xiong B: Identification of two novel biomarkers of rectal carcinoma progression and prognosis via co-expression network analysis. Oncotarget 8: 69594-69609, 2017.

23. Yip AM and Horvath S: Gene network interconnectedness and the generalized topological overlap measure. BMC Bioinformatics 8: $22,2007$.

24. Hui L, Zhang J, Ding X, Guo X and Jang X: Identification of potentially critical differentially methylated genes in nasopharyngeal carcinoma: A comprehensive analysis of methylation profiling and gene expression profiling. Oncol Lett 14: 7171-7178, 2017. 
25. Chatraryamontri A, Breitkreutz BJ, Oughtred R, Boucher L, Heinicke S, Chen D, Stark C, Breitkreutz A, Kolas N, O'Donnell L, et al: The BioGRID interaction database: 2015 update. Nucleic Acids Res 43 (Database Issue): D470-D478, 2015.

26. Keshava Prasad TS, Goel R, Kandasamy K, Keerthikumar S, Kumar S, Mathivanan S, Telikicherla D, Raju R, Shafreen B, Venugopal A, et al: Human protein reference database-2009 update. Nucleic Acids Res 37 (Database Issue): D767-D772, 2009.

27. Szklarczyk D, Franceschini A, Kuhn M, Simonovic M, Roth A, Minguez P, Doerks T, Stark M, Muller J, Bork P, et al: The STRING database in 2011: Functional interaction networks of proteins, globally integrated and scored. Nucleic Acids Res 39 (Database Issue): D561-D568, 2011.

28. Lopes CT, Franz M, Kazi F, Donaldson SL, Morris Q and Bader GD: Cytoscape Web: An interactive web-based network browser. Bioinformatics 26: 2347-2348, 2010.

29. Gene Ontology Consortium: Gene ontology consortium: Going forward. Nucleic Acids Res 43 (Database Issue): D1049-D1056, 2015.

30. Kanehisa M, Sato Y, Kawashima M, Furumichi M and Tanabe M: KEGG as a reference resource for gene and protein annotation. Nucleic Acids Res 44 (D1): D457-D462, 2016.

31. Huang da W, Sherman BT and Lempicki RA: Systematic and integrative analysis of large gene lists using DAVID bioinformatics resources. Nat Protoc 4: 44-57, 2009.

32. Hanson MA, Fareed MT, Argenio SL, Agunwamba AO and Hanson TR: Coronary artery disease. Prim Care 40: 1-16, 2013.

33. Patil DP and Kundu GC: LCK (lymphocyte-specific protein tyrosine kinase). Atlas Genet Cytogenet Oncol Haematol 9: 229-230, 2005

34. Pryshchep S, Goronzy JJ, Parashar S and Weyand CM: Insufficient deactivation of the protein tyrosine kinase lck amplifies T-cell responsiveness in acute coronary syndrome. Circ Res 106: 769-778, 2010.

35. Pelosi M, Di Bartolo V, Mounier V, Mège D, Pascussi JM, Dufour E, Blondel A and Acuto O: Tyrosine 319 in the interdomain B of ZAP-70 is a binding site for the Src homology 2 domain of Lck. J Biol Chem 274: 14229-14237, 1999.

36. Staudt LM, Rosenwald A, Wilson W, Barry TS and Wiestner A: ZAP-70 expression as a marker for chronic lymphocytic leukemia/small lymphocytic lymphoma (CLL/SLL). US Patent 7,981,610 B2, Filed December 12, 2007; issued July 19, 2011.

37. Flego D, Liuzzo G, Weyand CM and Crea F: Adaptive immunity dysregulation in acute coronary syndromes: From cellular and molecular basis to clinical implications. J Am Coll Cardiol 68 : 2107-2117, 2016
38. Li H, Zuo X, Ouyang P, Lin M, Zhao Z, Liang Y, Zhong S and Rao S: Identifying functional modules for coronary artery disease by a prior knowledge-based approach. Gene 537: 260-268, 2014.

39. Tachibana M, Sugimoto K, Fukushima T and Shinkai Y: Set domain-containing protein, G9a, is a novel lysine-preferring mammalian histone methyltransferase with hyperactivity no specific selectivity to lysines 9 and 27 of histone H3. J Biol Chem 276: 25309-25317, 2001

40. Zhang QJ and Liu ZP: Histone methylations in heart development, congenital and adult heart diseases. Epigenomics 7: 321-330, 2015.

41. Papait R, Serio S, Pagiatakis C, Rusconi F, Carullo P, Mazzola M, Salvarani N, Miragoli M and Condorelli G: Histone methyltransferase G9a is required for cardiomyocyte homeostasis and hypertrophy. Circulation 136: 1233-1246, 2017.

42. Thienpont B, Aronsen JM, Robinson EL, Okkenhaug H, Loche E, Ferrini A, Brien P, Alkass K, Tomasso A, Agrawal A, et al: The H3K9 dimethyltransferases EHMT1/2 protect against pathological cardiac hypertrophy. J Clin Invest 127: 335-348, 2017.

43. Bremer S, Rootwelt $\mathrm{H}$ and Bergan S: Real-time PCR determination of IMPDH1 and IMPDH2 expression in blood cells. Clin Chem 53: 1023-1029, 2007.

44. Xu Y, Zheng Z, Gao Y, Duan S, Chen C, Rong J, Wang K, Yun M, Weng H, Ye S and Zhang J: High expression of IMPDH2 is associated with aggressive features and poor prognosis of primary nasopharyngeal carcinoma. Sci Rep 7: 745, 2017.

45. Zhou L, Xia D, Zhu J, Chen Y, Chen G, Mo R, Zeng Y, Dai Q, $\mathrm{He} \mathrm{H}$, Liang Y, et al: Enhanced expression of IMPDH2 promotes metastasis and advanced tumor progression in patients with prostate cancer. Clin Transl Oncol 16: 906-913, 2014.

46. Gingras AC, Caballero M, Zarske M, Sanchez A, Hazbun TR, Fields S, Sonenberg N, Hafen E, Raught B and Aebersold R: A novel, evolutionarily conserved protein phosphatase complex involved in cisplatin sensitivity. Mol Cell Proteomics 4: $1725-1740,2005$.

47. Eleftheriadou O, Longman MR, Boguslavskyi A, Ryan A, Wadzinski BE, Shattock MJ and Snabaitis AK: Expression of type 2a protein phosphatases in cardiac health and disease. Heart 100: A16, 2014.

48. Eleftheriadou O, Boguslavskyi A, Longman MR, Cowan J, Francois A, Heads RJ, Wadzinski BE, Ryan A, Shattock MJ and Snabaitis AK: Expression and regulation of type $2 \mathrm{~A}$ protein phosphatases and alpha4 signalling in cardiac health and hypertrophy. Basic Res Cardiol 112: 37, 2017.

This work is licensed under a Creative Commons Attribution-NonCommercial-NoDerivatives 4.0 International (CC BY-NC-ND 4.0) License. 